\title{
Agents for Searching Rules in Civil Engineering Data Mining
}

\author{
Janusz Kasperkiewicz and Maria Marks \\ Institute of Fundamental Technological Research, \\ Polish Academy of Sciences, \\ 21 Świętokrzyska Str., 00-049 Warsaw, Poland \\ \{J .Kasperkiewicz, M.Marks\}@ippt.gov.pl
}

\begin{abstract}
The software agents are applied for a remote search of information. It seems natural that to analyse such information machine learning routines should be built-in into an agent system. After finding and processing the data the generated rules will be evaluated by means of so called interestingness measures, and only the best rules should be returned to the user.

The paper presents situation in civil engineering data processing, as a suggestion for designers of intelligent software tools, to work out difficult but much needed procedures that should be implemented into autonomous agent system, intended for retrieving special kind of information searched for example by materials technologists.

A simple architecture for an agent system is suggested without, however, getting into any technical details on how the elements of such system should be constructed.
\end{abstract}

Keywords: machine learning, data mining, software agents, engineering databases.

\section{Introduction}

Generally speaking the agents are to reduce the workload of their users, but there is a diversity of kinds of information interesting for different users. Many users are satisfied only with a task of simply finding appropriate documents, like text files, Internet sites, data tables, images, audio or video files, which are dedicated to specific topics. Introducing agents able not only to find but also to process the data might immensely increase the possibilities of remote knowledge search.

An agent is intelligent, autonomous code that can be send out on a mission, 1]. It can work in a static way, being located at the local system, or it may be mobile, working after being embedded in a remote machine. Agents may be static or mobile, acting in a collaborative way or separately, but their crisp definition is difficult and a classic paper of 1996, [2, was pointing out a general lack of precision in software agents taxonomy. It seems that the situation in this respect is not much different today. In the description which follows the case of static agent is assumed.

M. Bubak et al. (Eds.): ICCS 2008, Part III, LNCS 5103, pp. 702-711, 2008.

(C) Springer-Verlag Berlin Heidelberg 2008 
Machine learning (ML) programs are not agents, but it seems natural that they might and should be built in into an agent system. ML programs usually are constructed for purposes more sophisticated than simple finding of information sources. In our investigations we are using them mainly for searching rules representing knowledge about technological processes under inspection. An example here can be a process of selecting proper mix of components in a composite material.

A concept of an agent for engineering data search will be explained on an example. An agent could be a personification of an Internet user, who from the computer terminal is searching, e.g. around WWW, or some protected network, or the whole Internet, or only the single computer of the user, for a particular kind of information. After the information source is found, (e.g. a report with experimental results), it is usually downloaded by the user from the remote system, before it can be analysed to extract the knowledge in form of statements, (hypotheses, rules, generalisations). Generation of rules is performed by the ML programs. A rule of practical importance for a civil engineer can be for example an information, in form of a relation, what amounts of certain components of a concrete mix correspond to a desired quality of the resulting hardened concrete material.

In our group at IPPT1 1 we are using various kinds of AI tools, such as ANNs, (Artificial Neural Networks), but especially ML methods for prediction of rules. Important in our investigations were the methods developed by Ryszard Michalski and his team at George Mason University; programs: AQ15, AQ19, AQ21. We were also comparing them with various other ML algorithms.

The present paper is to indicate certain important characteristics of agent type programs that might bring most needed results. It is assumed that the agent will generate the rules concerning certain process; the details of creation of association rules, of the decision trees, etc., are not discussed in this text. Also technical questions such as the protection of the data or the robustness of the agent, etc., are not discussed here.

\section{Machine Learning Tools}

Learning systems have the ability of setting values of their certain internal parameters, of some preset, abstract algorithm, in a way that will minimize difference between the internal results and the external control values. The values can be scalars, vectors or categories. If during the training the configuration of the internal parameters tends to a certain steady state, such state represents the knowledge of the system about the process under consideration.

A generalization of the learned knowledge can be a black-box type tool, like in case of ANNs, (Artificial Neural Networks), or a set of various concepts, (rules, hypotheses), expressed in form of logical expressions, such as a proposition: $A \rightarrow B$.

${ }^{1}$ IPPT - Institute of Fundamental Technological Research, Polish Academy of Sciences. 
This expression represents an inference containing an antecedent $(A)$ and the consequent $(B)$. The antecedent $A$ may represent a larger set of simple conditions on the components of the input and $B$ represents output conditions, typically it is a designation of the class, of a certain category.

The symbol $A$ represents some concept, which is a conjunction, (or a complex), of simple, or atomic conditions, called selectors, described by numerical or nominal attributes of the dataset. The selectors are marked by square brackets, $([\ldots])$. A few examples of selectors are: $[x \leq 12],[y>2.37],[z \in$ $(1.30,1.77\rangle]$, in case of only numerical variables, and $\left[v \in\right.$ 'red $^{\prime},{ }^{\prime}$ blue , $^{\prime}$ yellow $]$, $\left[w \notin ' A^{\prime},{ }^{\prime} D^{\prime},{ }^{\prime} K^{\prime},{ }^{\prime} L^{\prime},{ }^{\prime} R^{\prime}\right]$ - where only nominal variables appear.

For any record in the training or testing datasets the hypothesis in form of the proposition $A \rightarrow B$ can be either true or false. In rare cases it can also be undefined.

A task for ML programs is to propose to the user rules of the type described above, selected from the collection of the trainee set of training hypotheses, [3]. The selection should correspond to a possibly low error rate of the power of a concept in predicting its consequences, characterizing the dataset under consideration. A finally selected rule might be for example:

$$
\text { if }[y>2.37] \text { and }\left[\left[v={ }^{\prime} \text { red }^{\prime}\right] \text { or }\left[v={ }^{\prime} \text { yellow' }\right]\right] \text { then }[\text { class }=\text { ClassIII }]
$$

The requirements concerning the formatting of the database are slightly different for different ML programs, but it is relatively easy to translate an input script created for one system to another one.

There is a number of different Machine Learning programs available. Many of these are more or less for free, like AQ15, AQ19, AQ21, C4.5, WinMine, Gradestat, WEKA, Rosetta, (a rough sets toolbox), 4, 5, 6, 7].

There are many commercial data mining programs, as was described e.g. in [8, 9. Examples are: DataCruncher, IBM Intelligent Miner, MineSet, CART, See5, WizRule, Magnum Opus, etc. Various elements of rules searching from database examples are also available now in large commercial packages dedicated to statistics and data mining, like Oracle, Statistica, SPSS or SAS.

In civil engineering there is a hypothetical possibility of combining various soft computing tools into a one system, [10, 11, 12, but so far it is still only the concept that needs much further programming work.

\section{Civil Engineering Data Bases}

Civil engineering experimental data are scattered around various sources, such as technical papers, reports, books, standards and instructions, also special databases, which are properties of laboratories or production managers. Many of such sources are available only in a paper form, so to transfer them into electronic form considerable additional work would be needed. It is assumed in this paper that there is a certain digital data environment accessible, in which the agent will function. This may be simply a selected directory in the computer of the user, the contents of a network of connected computers, or any library that may be opened in the Internet. 
There are different types of engineering data. These are descriptive databases concerning composition of concrete and similar composite materials, data concerning various diagnostic problems, data enabling only the quantitative predictions, data allowing only for classification, etc. For example the properties of concrete depend on composition of the original concrete mix, on certain elements of its mixing technology, on its curing and protection, its age, sometimes on its whole history. The properties of concrete can be characterized by attributes in form of numbers, (like: strength, density, amounts of cement, water or additives), and in form of categories, (like: 'basalt', 'limestone', 'PFA1', 'PFA2', etc.)

The fields of investigation may be properties of materials, diagnostics of the quality of materials, diagnostics of whole engineering structures. In any case for processing by ML algorithms, (Machine Learning), the data must be properly formatted, so that the program would not encounter any unannounced values.

A very simple example of a structure of a database prepared for generation of rules for civil engineering purposes is shown in Table 1. The attributes of the records can, in the simplest case, be of two different types: numerical or nominal. There is number of different other attribute types, such as date or string, which are not discussed here. Any attribute in a database record may also have an unknown value, (a symbol '?' is usually applied).

Table 1. An example of a simple structure of a formatted database

\begin{tabular}{|c|c|c|c|c|}
\hline name & type & action & min & max \\
\cline { 4 - 5 } & & & or - list of legal values \\
\hline \hline No & numeric & ignore & 1 & 455 \\
nrpomiaru & numeric & ignore & 1 & 502 \\
lzdH & numeric & active & 394 & 660 \\
lzdM & numeric & active & 461 & 833 \\
lzdL & numeric & active & 886 & 1027 \\
senH & numeric & active & 35 & 71 \\
senM & numeric & active & 62 & 286 \\
senL & numeric & active & 351 & 606 \\
sazH & numeric & active & 14 & 33 \\
sazM & numeric & active & 23 & 178 \\
sazL & numeric & active & 103 & 248 \\
\cline { 4 - 5 } phase & nominal & ignore & \multicolumn{2}{|c|}{$\mathrm{cp}, \mathrm{a}, \mathrm{v}$} \\
\cline { 3 - 4 } HV & numeric & ignore & \multicolumn{2}{|c|}{56} \\
\cline { 3 - 4 } class & nominal & active & $\mathrm{A} 0, \mathrm{~A} 15 \mathrm{~K}, \mathrm{~A} 15 \mathrm{~T}, \mathrm{~A} 30 \mathrm{~K}, \mathrm{~A} 30 \mathrm{~T}$ \\
\hline
\end{tabular}

A formatted database can be conceived as a matrix in which the rows represent records and the columns represent attributes.

All the information on the structure of a database like in this example are needed to organise properly the ML processing of the dataset. Not all the attributes must be taken into account during the final rule generation. Working on a number of attributes bigger than the necessary minimum might result in an unnecessary noisiness of the data. The designation 'ignore' in the example 
above, ('ignore', as opposite to 'active'), concerns the attributes that were excluded from the calculations in the example in Chapter 5 .

There are various actions that an agent should do. A difficult and important task to be fulfilled by an agent is after identification of the source, (e.g. finding in the Internet a paper dedicated to some type of concrete), to evaluate this source from the point of view of "useful data", (i.e. whether it contains the kind of data that this particular agent will be able to recognise and format), to decide how the data will be procured and transformed, and - if needed - also imported to the agent's home site.

Not discussed here are quite obvious but more particular tasks for agent system, for example optical character recognition in case of the PDF documents available in form of images, recognition of different decimal systems, unification of descriptions, identification, proper understanding and translation in case of foreign languages, etc.

\section{The Concept of Interestingness}

The ML programs often produce for a given dataset many, even hundreds of particular rules. For a user who is looking for rules as simple as typical empirical formulae proposed over the ages by the human experts, the rules produced typically by a ML program are of unequal value, many practically useless. Worth further attention may be only some of them - those "most interesting" ones.

The concept of interestingness has appeared in data mining literature mainly in the last decade, (perhaps one of the first uses of the term was in 1995, by Silberschatz and and Tuzhilin, as cited in [13]). It concerns the relative importance of any rules conceived by people, but mainly of the rules generated by machine learning algorithms. The meaning of the term interestingness is slightly imprecise, because the value of a rule depends naturally on the point of view of the user.

There are more than 30 different interestingness measures discussed in the literature; cf. for example [13, 14, 15, 16. They are mostly constructed by algebraic operations on a set of primary measures of amounts of records in a database under consideration, (in certain texts, depending on the field of interests of the author, instead of the term records used is the word transactions; e.g. [17]). And some investigators apply their own measures concerning the quality of the rules, without referring at all to the notion of interestingness, (e.g. [5, 7, 18]).

With association rules in form of $A \rightarrow B$, (the antecedents in $A$ and consequent or target class $B$, being conjunctions of simple rules or selectors of attributes), and $n$ being a total number of records in a database, the primary measures are: $n_{a}, n_{b}, n_{a b}$ and $n_{a \neg b}$, meaning numbers of records matching the conditions, respectively, of $A, B, A \cap B, A \cap \neg B$, (here $\neg B$ means: NOT B).

Examples of four simplest and most typical interestingness measures are: Support, Confidence, Conviction and Lift2. They are, respectively, defined by formulae:

${ }^{2}$ the same name Lift corresponds to a different operator in See5, although there is close linear correlation between the results of the both formulae. 


$$
\begin{gathered}
\text { support }=\left(n_{a}-n_{a \neg b}\right) / n, \quad \text { confidence }=1-n_{a \neg b} / n_{a}, \\
\text { conviction }=n_{a} n_{\neg b} /\left(n n_{a \neg b}\right), \quad \text { lift }=n\left(n_{a}-n_{a \neg b}\right) /\left(n_{a} n_{b}\right) .
\end{gathered}
$$

Among the interestingness measures discussed in the literature rarely mentioned is a measure characterizing the simplicity of a rule. For example very important may be the difference whether the rule is composed of only 1, 2 or 3 selectors, and not - e.g. - 20 selectors or more. The issue is treated in AQ21, where a special function is introduced that can be used to minimize the complexity of learned rules.

In the present paper it is proposed to introduce an additional interestingness measure called Simplicity, calculated as an inverse of the number of selectors on the antecedents' side of the rule in question.

In this way a vector of 5 interestingness measures designated respectively as: sup., conf., conv., lift and simpl., presents a compact set of parameters, enabling the user a quick evaluation and comparison of the applied ML procedures.

\section{Experiments}

The particular experiments with ML procedures discussed in what follows were dedicated to a case of data collected during the microindentation tests on hardened concrete, using a Vickers indenter, and recording acoustic emission signals, which were subsequently processed by wavelet transformation, [19. The database of about 300 records contained, among others, 9 columns of numbers characterising the acoustic emission signal, (AE), on selected frequency and magnitude levels. The structure of the database was presented in the Table 1 above.

The task of ML programs were to discover from the AE data the rules allowing recognizing which records correspond to concrete containing certain additives, (like fly ash or PFA), and which ones are without those additives. The issue may be of importance in case of forensic analysis problems in construction of questionable quality. The presence of additives in the experiment was identified by a class code, (there were three levels of the additive content: 0, 15 and 30 percent, and two additive sources: $\mathrm{K}$ and $\mathrm{T}$; cf. the bottom raw of the Table 11).

In search of the rules applied were mainly four ML tools: See5, AQ19, WEKA and AQ21, 5, 6, 7, 18. A number of different rules were obtained from different methods. In many cases the primary measures concerning numbers of records supporting rules generated by the system could be evaluated directly from the accuracy statistics indices built-in into programs. In other cases they were calculated manually using MS Excel.

Selected results in form of rules, the primary measures and the corresponding interestingness measures are presented in Table 2.

The database from which the numbers were taken in the Table 1 was of 239 records, $(n=239)$, with the numbers, $\left(n_{b}\right)$, of records in five different classes A0, A15K, A15T, A30K and A30T being, respectively, 80, 60, 62, 63 and 64. The numbers $n_{a}, n_{a b}$ and $n_{a \neg b}$ were either taken from the ML programs or were counted in Excel. 
Table 2. Examples of interestingness measures obtained using four ML programs (the cases of conv=max correspond to rules in which there were no erroneous predictions)

\begin{tabular}{|c|c|c|c|c|c|c|c|c|}
\hline & $n_{a}$ & \multicolumn{2}{|c|}{\begin{tabular}{|l|l|}
$n_{a b}$ & $n_{a \neg b}$ \\
\end{tabular}} & sup & \multicolumn{2}{|c|}{\begin{tabular}{|l|l|} 
conf & conv \\
\end{tabular}} & 1111 & $1 \mathrm{im}$ \\
\hline \multicolumn{9}{|c|}{$\overline{\text { Rules according to See5 }}$} \\
\hline$\rightarrow$ Class $=A 0$ & 229 & 78 & 151 & 0.24 & 0.34 & 1.15 & 1.06 & 1.00 \\
\hline$[$ sazH $>18][$ sazL $<=167]$ & 108 & 41 & 67 & 0.12 & 0.38 & 1.30 & 1.57 & 0.50 \\
\hline$[\operatorname{sen} L>400][\operatorname{saz} H<=17]$ & 16 & 14 & 2 & 0.04 & 0.88 & 6.49 & 3.77 & 0.50 \\
\hline$\rightarrow$ Class $=A 15 K$ & 100 & 39 & 61 & 0.12 & 0.39 & 1.34 & 1.75 & 1.00 \\
\hline$[l z d H>486][\operatorname{sen} H>54]$ & 35 & 16 & 19 & 0.05 & 0.46 & 1.51 & 2.05 & 0.50 \\
\hline$[\operatorname{sen} H>47][\operatorname{sen} H<=54]$ & 62 & 21 & 41 & 0.06 & 0.34 & 1.22 & 1.43 & 0.50 \\
\hline \multicolumn{9}{|c|}{ Rules from AQ19 } \\
\hline $\begin{array}{l}{[l z d H=510 . .578][l z d M=697 . .800]} \\
{[l l z d L=976 . .1007,1027][\operatorname{sen} H=39 . .42]} \\
{[\operatorname{senL}=351 . .393]}\end{array} \stackrel{\rightarrow \text { Class }=A 0}{ }$ & 51 & 35 & 16 & 0.11 & 0.69 & 2.41 & 2.14 & 0.20 \\
\hline $\begin{array}{l}{[l z d H=486 . .555,571][l z d M=655 . .748,807]} \\
{[l z d L=950 . .1005][\operatorname{sen} H=39 . .41]} \\
{[\operatorname{sen} L=379 . .507]}\end{array} \quad \rightarrow$ Class $=A 15 T$ & 28 & 21 & 7 & 0.06 & 0.75 & 3.25 & 3.23 & 0.20 \\
\hline $\begin{array}{l}{[l z d H=394 . .531,633][l z d L=950 . .1006]} \\
{[\operatorname{sen} H=42 . .57][\operatorname{sen} M=99 . .286][\operatorname{saz} M=47 . .148]} \\
{[\operatorname{sazL}=113 . .145,177 . .195] \quad \rightarrow \text { Class }=A 30 T}\end{array}$ & 19 & 16 & 3 & 0.05 & 0.84 & 5.10 & 3.49 & 0.17 \\
\hline \multicolumn{9}{|c|}{ Rules from WEKA, (classifier PART) } \\
\hline $\begin{array}{l}{\left[\begin{array}{l}\operatorname{sen} H>47][l z d L<=942] \\
{[l z d H>465]}\end{array}\right.} \\
{[l z H=1}\end{array}$ & 9 & 8 & 1 & 0.02 & 0.89 & 7.36 & 3.99 & 0.33 \\
\hline$[\operatorname{sen} H>47][l z d L>974][$ sazH $>21] \rightarrow$ Class $=A 30 T$ & 10 & 10 & 0 & 0.03 & 1.00 & $\max$ & 4.14 & 0.33 \\
\hline \multicolumn{9}{|c|}{ "Rules from AQ21 } \\
\hline 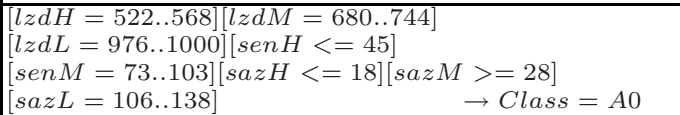 & 16 & 16 & $\overline{0}$ & 0.05 & 1.00 & $\max$ & 3.11 & 0.13 \\
\hline$\rightarrow$ Class $=A 15 K$ & 135 & 48 & 87 & 0.15 & 0.36 & 1.27 & 1.59 & 1.00 \\
\hline$\rightarrow$ Class $=A 15 T$ & 132 & 44 & 88 & 0.13 & 0.33 & 1.22 & \begin{tabular}{|l|l|}
1.44 \\
\end{tabular} & 1.00 \\
\hline
\end{tabular}

The numbers in bold print in the last columns of the Table 2 correspond to best results from the point of view of a given interestingness measures. The limit values are 1 in case of Support, Confidence and Simplicity measures. There are no practical limits in cases of Conviction and Lift, or, to be more precise the maximum number that can appear there depends on the size of the database under investigation.

As can be seen the rules obtained by the programs have limited effectiveness. The rules are either of low support even being quite reliable, or are of low accuracy, (many false predictions), or are very complicated, (e.g. 8 selectors in a rule).

It should be added, that the recognition of the data by ML programs was generally rather good. It is obvious that effectiveness of the whole set of rules generated by any ML program used together as a whole collection of formulae may be very effective. For example by applying a whole set of 60 rules obtained by one of the programs, (it was See5), quite a satisfying confusion matrix was obtained, as shown in Fig. 17a. Confusion matrix displays how the ML system assigns the records to their respective classes. When in the next run selected was the Boost-10 trials option the results were almost ideal - Fig. 1 b.

Similar results were obtained also using the other ML programs. The good recognition, however, is an effect of applying a kind of voting procedures, and 


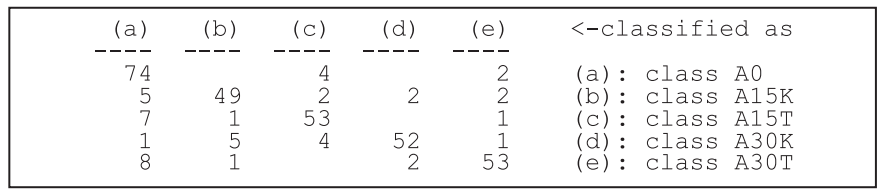

\begin{tabular}{|c|c|c|c|c|c|}
\hline (a) & (b) & (c) & $(d)$ & (e) & $<-$ classified as \\
\hline 80 & $\begin{array}{r}60 \\
1\end{array}$ & 62 & 62 & 64 & $\begin{array}{l}\text { (a): class A0 } \\
\text { (b): class A15K } \\
\text { (c): class A15T } \\
\text { (d) } \text { class A30K } \\
\text { (e) } \text { class A30T }\end{array}$ \\
\hline
\end{tabular}

Fig. 1. Confusion matrices obtained on the same dataset with program See5: (a) default settings, (b) - after selecting the option: Boost (10 trials)

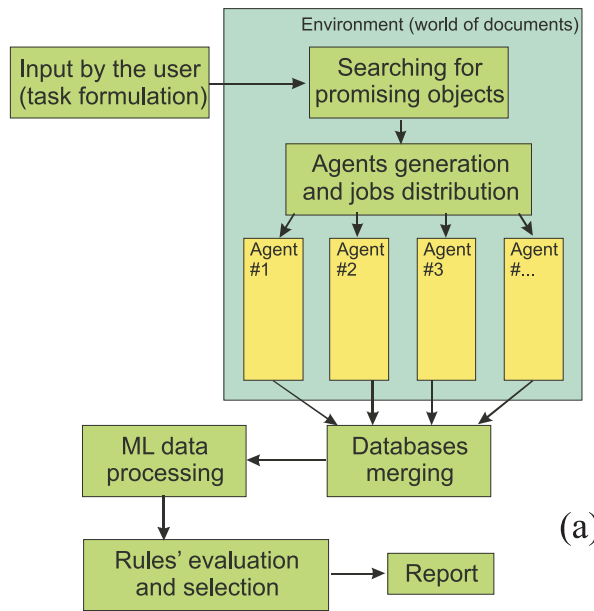

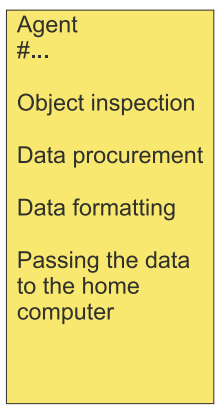

(a)

(b)

Fig. 2. Proposed architecture - (a) of the agent system, (b) of the sub-agent

there are no simpler formula resulting from such calculation. And these possibly simple formulae are what is really needed by engineers.

The selected rules presented in the first column of Table 2 were obtained at a default settings in cases of See5 and AQ19, applying the classifiers PART in case of WEKA, and applying the PD mode, (Pattern Discovery), in case of AQ21.

\section{Conclusions}

It seems that the ambitions presented in this paper seem to be rather farreaching. It would be however really advantageous to strive towards creating an agent system able to realize proceedings described above. Or at least some elements of it, as these also might be helpful to a human researcher. 
After being activated the agent, (agents), should start looking for appropriate data sources, to process what is found, and return finally a number of rules, each accompanied with an interestingness measures vector, as defined in the previous chapter. For the beginning, however, it would be important to have at least one agent that could identify which papers, from a list of hundreds of titles, contain the tables of interesting experimental results.

In Fig. 2 shown is what seems to be a possible architecture for such an agent system.

The input by the user would be some characterization of the field of search, for example by typing-in a number of keywords, (e.g.: "concrete, additives, admixtures, silica, PFA"; as previously this is an example from the field of concrete-like composite materials), and a proposal of a list of expected headings of the columns in the tables presenting the features aimed at during the search. Such list would have to contain as many alternatives as the user can think of. For example: "w, water, c, cement, silica, silica fume, sup, superplasticiser, aggregate, CA, FA, air entrainment, strength, compression strength, density, fc28", (as can be seen the same attributes may have different symbols in different data sources).

After receiving the results from such system the user is thereupon to decide which results are better from others. The rules obtained by different procedures are mutually supplementing, to combine the results, however, a human action would be needed, so the question of how to combine the rules is not discussed here further.

There is a general observation from the experiments performed on actual experimental data using different ML tools that very often the resulting set of rules was either too large, (e.g. 100 rules), or the rules were too complex, (more than $3 \div 4$ selectors), or they had too low the support or too low the accuracy. The results like those in Table 2 will allow the user to concentrate on only the most important results of the search.

The problem of finding optimal rule is a really multi-criteria task. The user in the presented example could support his or her estimate of the resulting rulsets by the five components of the interestingness vector. Later on the user will be able to work out the position to recognise the value of the search by the first look into the results.

Acknowledgments. This work was supported by Projects No. R04 01301 and No. 4 T07E 036 30, sponsored by The Ministry of Science and Higher Education, Warsaw, Poland, (MNiSW), to which the authors are grateful.

\section{References}

[1] Ouderkirk, J.: Technical services task assignment: from macros to collection management intelligent agents. The Journal of Academic Librarianship 25, 397-401 (1999)

[2] Nwana, H.S.: Software agents: An overview. Knowledge Engineering Review 11(2), 205-244 (1995)

[3] Cichosz, P.: Learning systems. WNT, Warszawa (in Polish) (2000) 
[4] Gradestat - a statistical program for exploratory data analysis, http://gradestat.ipipan.waw.pl/

[5] Michalski, R.S., Kaufman, K.A.: The AQ19 system for machine learning and pattern discovery: A general description and user's guide (2001)

[6] Witten, I.H., Frank, E.: Data Mining: Practical Machine Learning Tools and Techniques, 2nd edn. Morgan Kaufmann Series in Data Management Sys. Morgan Kaufmann, San Francisco (2005)

[7] Wojtusiak, J.: AQ21 user's guide. Technical Report MLI 04-3, P 04-5 (2004)

[8] Elder, J.I., Abbott, D.: A comparison of leading data mining tools. In: Fourth International Conference on Knowledge Discovery \& Data Mining (1998)

[9] King, M., Elder, I.J., Gomolka, B., Schmidt, E., Summers, M., Toop, K.: Evaluation of fourteen desktop data mining tools. In: IEEE International Conference on Systems, Man, and Cybernetics, vol. 3, pp. 2927-2932 (1998)

[10] Alterman, D., Kasperkiewicz, J.: Evaluating concrete materials by application of automatic reasoning. Bulletin of the Polish Academy of Sciences. Technical Sciences 54(4), 352-362 (2006)

[11] Alterman, D.: Evaluation of concrete materials by automatic reasoning. IPPT PAN, Polish Academy of Sciences (in Polish) (manuscript, 2005)

[12] Kasperkiewicz, J., Alterman, D.: Holistic approach to diagnostics of engineering materials. Computer Assisted Mechanics and Engeneering Sciences 14, 197-207 (2007)

[13] Vaillant, B.: Mesurer la qualité des regles d'association. Etudes formelles et expérimentales. l'École Nationale Supérieure des Télécommunications de Bretagne en habilitation conjointe avec l'Université de Bretagne Sud (2006)

[14] Huynh, X., Guillet, F., Briand, H.: ARQAT: an exploratory analysis tool for interestingness measures. In: Janssen, J. (ed.) 11th international symposium on Applied Stochastic Models and Data Analysis (ASMDA 2005), Brest, France, May 17-20, 2005, pp. 334-344 (2005)

[15] Lallich, S., Vaillant, B., Lenca, P.: A probabilistic framework towards the parameterization of association rule interestingness measures. Methodology and Computing in Applied Probability 9(3), 447-463 (2007)

[16] Lenca, P., Meyer, P., Vaillant, B., Lallich, S.: On selecting interestingness measures for association rules: User oriented description and multiple criteria decision aid. European Journal of Operational Research 127(2), 610-626 (2008)

[17] McGarry, K.: A survey of interestingness measures for knowledge discovery. Knowl. Eng. Rev. 20(1), 39-61 (2005)

[18] Rulequest research 2007, See5: An informal tutorial http://www.rulequest.com/see5-win.html

[19] Kasperkiewicz, J.: On a possibility of structure identification by microindentation and acoustic emission. In: de Miguel, Y., Porro, A., Bartos, P. (eds.) 2nd International Symposium on Nanotechnology in Construction, NICOM 2, Bilbao, Spain, pp. 151-159. RILEM Publications S.A.R.L (2006) 